\title{
Economic arguments for the immediate management of moderate-to- severe obstructive sleep apnoea syndrome
}

\author{
N. Pelletier-Fleury*, N. Meslier*, F. Gagnadoux\#, C. Person*, D. Rakotonanahary\#, H. Ouksel*, \\ B. Fleury", J-L. Racineux*
}

Economic arguments for the immediate management of moderate-to-severe obstructive sleep apnoea syndrome. N. Pelletier-Fleury, N. Meslier, F. Gagnadoux, C. Person, D. Rakotonanahary, H. Ouksel, B. Fleury, J-L. Racineux. (C)ERS Journals Ltd 2004.

ABSTRACT: The objective of this study was to measure the impact of a 6-month delay in the diagnosis and treatment of patients with moderate obstructive sleep apnoea syndrome (OSAS) (apnoea/hypopnoea index (AHI) $<30)$ or severe OSAS (AHI $\geqslant 30$ ) on daytime sleepiness, cognitive functions, quality of life and healthcare expenditure (hospitalisations, medical visits, complementary tests, biological tests and drug prescriptions). In addition, this study aimed to analyse the incremental cost effectiveness ratios related to daytime sleepiness or quality of life following immediate introduction of treatment in these two populations.

This study was conducted as a multicentre randomised controlled trial and carried out at two teaching hospitals in France. A total of 171 patients were followed for 6 months, with 82 patients randomised to group 1 "immediate polysomnography" and 89 in group 2 "polysomnography within 6 months".

Patients with severe OSAS were deprived of a significant improvement of their daytime sleepiness $(5.1 \pm 5.0$ at the Epworth Sleepiness Scale score in group 1 versus 0.2 \pm 3.4 in group 2) and quality of life (12.4 \pm 13.3 at the Nottingham Health Profile score in group 1 versus $0.7 \pm 10.1$ in group 2) during the waiting time. The impact of delayed management in subjects with less severe OSAS only concerned daytime sleepiness (1.9 \pm 3.3 in group 1 versus $0.3 \pm 4.3$ in group 2$)$. Delayed treatment did not affect cognitive functions or healthcare expenditure regardless of the severity of the disease. Incremental cost effectiveness ratios related to rapid introduction of treatment were significantly lower in the patients with more severe OSAS.

These results provide fairly clear medical and economic arguments in favour of early management of patients with more severe forms of obstructive sleep apnoea syndrome.

Eur Respir J 2004; 23: 53-60.
*Unité de Sommeil, Service de Pneumologie, CHU d'Angers, Angers, and ${ }^{\#}$ Unité de Sommeil, Service de Pneumologie, CHU Saint Antoine, Paris, France.

Correspondence: N. Pelletier-Fleury Institut National de la Santé et de la Recherche Médicale U537 (Centre de Recherches en Economie et Gestion Appliquées à la Santé) 80 rue du Général Leclerc

94276 Le Kremlin-Bicêtre Cedex

France

Fax: 33146713270

E-mail: fleury@kb.inserm.fr

\section{Keywords: Apnoea}

continuous positive airway pressure

costs

economic evaluation

\section{Received: June 132003}

Accepted after revision: September 42003

This study was supported by the Programme Hospitalier de Recherche Clinique ${ }^{\circ} 9706$
The growing demand for the diagnosis of obstructive sleep apnoea syndrome (OSAS) by nocturnal polysomnography (PSG) performed in a sleep laboratory leads to longer waiting lists for this examination and for therapeutic management of patients by continuous positive airway pressure (CPAP). In France, as in other parts of Europe, patients wait an average of 6 months to $1 \mathrm{yr}$ in most public hospital sleep centres. To overcome this situation, it would be necessary to increase the healthcare supply, either by increasing the number of beds per sleep laboratory or by increasing the number of sleep laboratories. However, such a solution would appear to be unfeasible in view of the limited resources devoted to hospitals. Other technical solutions have been proposed including split-night PSG [1, 2], which would halve the number of PSGs performed in sleep laboratories, outpatient PSG [3, 4], or PSG telemonitored from a sleep laboratory [5], which would replace PSG in the laboratory. The respective advantages of these techniques remain controversial. Some studies have compared the effectiveness and costs of each of these replacement solutions to those of sleep laboratory PSG with extremely variable results $[6,7]$.

The assumption underlying the search for these various replacement solutions for sleep laboratory PSG, and which explains the abundance of scientific studies in this field, is that a delay in the diagnostic and therapeutic management of patients with OSAS may present one or several disadvantages for the patient or for the community. Recent studies have demonstrated an improvement of the quality of life of patients treated with CPAP under certain conditions [8-18], while other studies have shown a higher healthcare expenditure in patients with untreated OSAS [19-25]. However, no study has ever specifically analysed the consequences of this delayed management, in terms of costs and changes of individual health status during the waiting period.

The objectives of this randomised clinical trial were first, to measure the impact of a 6-month delay in the diagnosis and treatment of patients with moderate OSAS (apnoeahypopnoea index 10-30) or severe OSAS (apnoea/hypopnoea index $(\mathrm{AHI}) \geqslant 30$ ) on daytime sleepiness, cognitive function, quality of life and healthcare expenditure and secondly, to analyse the incremental cost effectiveness ratios related to daytime sleepiness or quality of life following immediate introduction of treatment in these two populations of OSAS. 


\section{Materials and methods}

\section{Patient inclusion criteria}

All patients aged 18-70 yrs attending the sleep unit of the Respiratory Medicine Depts of Angers Hospital and SaintAntoine Hospital in Paris for clinical suspicion of OSAS based on a combination of snoring and excessive daytime sleepiness were invited to participate in the trial.

\section{Patient exclusion criteria}

Patients presenting an indication for emergency treatment by CPAP (unstable coronary disease, daytime sleepiness interfering with car driving, especially in professional drivers) were excluded from the trial. The other exclusion criteria were chronic respiratory failure (arterial oxygen tension $\leqslant 7.315 \mathrm{kPa}$ $(\leqslant 55 \mathrm{mmHg}$ ) and/or carbon dioxide arterial tension $\geqslant 6.65 \mathrm{kPa}$ $(\geqslant 50 \mathrm{mmHg})$ and/or forced expiratory volume in one second (FEV1) $<1.5 \mathrm{~L} \cdot \mathrm{s}^{-1}$ and/or FEV1/vital capacity $<55 \%$ ), mental retardation, and patients unable to give their informed consent.

\section{Trial design}

This controlled, randomised, prospective, two-centre trial was conducted over a 2-yr period (1-yr inclusion period, 6 months of data collection for the last patients included, and 6 months of analysis). Consecutive subjects were randomly assigned to two different groups (a computed-generated

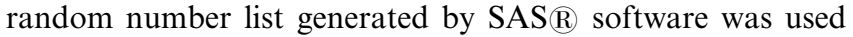
to assign patients to group 1 or group 2). In group 1, randomised to receive "immediate PSG", PSG was performed during the fortnight following the inclusion visit and treatment with CPAP was immediately proposed to all patients with an $\mathrm{AHI}>10$. These patients also received health and dietary advice (home diet if the body mass index was $>27 \mathrm{~kg} \cdot \mathrm{m}^{-2}$, avoidance of sedatives and alcohol consumption, adequate hours of sleep every night). In group 2, randomised to receive "PSG in 6 months", patients received the same health and dietary advice, an appointment for PSG was made 6 months after randomisation. In this group, patients with an AHI $\leqslant 10$ on the polysomnographic examination performed after a waiting time of 6 months were excluded from the analysis. This protocol allowed the authors to ensure comparative follow-up over 6 months of patients with OSAS treated by CPAP and patients waiting for treatment. The institutional committee on human research approved the study protocol and patients gave their written consent to participate in this trial after being informed about the practical modalities of the trial.

\section{Patients' follow-up}

On inclusion, all patients completed an individual questionnaire comprising anthropometric data, medical history, ongoing treatments, sleep habits and symptoms.

Assessment of sleepiness comprised of the following. 1) Epworth sleepiness scale (ESS) [26]. 2) Cognitive tests investigating attention and concentration (Trail Making Test A (TTA) and Trail Making Test B (TTB)). The parameter adopted was the time to complete TTB divided by the time to complete TTA. This ratio eliminated general speed variance (assessed by TTA time) from the time required for shifting (from letters to numbers and from numbers to letters) [27].
3) Quality of life was evaluated by the Nottingham Health Profile (NHP) [28]. The NHP includes 38 items exploring six dimensions of perceived health: energy, pain, sleep, physical mobility, emotional reactions and social isolation. For each item, the answer is either yes $(=1)$ or no $(=0)$. Each item was weighted and a final score was calculated for each dimension by adding the weighted answer for each item. For each dimension, the score ranged from zero (excellent perception of health) to 100 (very poor perception of health).

If analysis of the PSG results showed an AHI $>10$, treatment with CPAP was offered after a CPAP titration and habituation conducted over 2 nights. Manual titration of CPAP was performed in the sleep laboratory by a sleep technician. The progressive increase of the pressure was performed by steps of $1 \mathrm{cmH}_{2} \mathrm{O}$ until disappearance of apnoeas, hypopnoeas, flow limitations and snoring. Patients then returned home with the CPAP device. There was no wait with respect to provision of the CPAP equipment after the initial testing.

All patients included, in either group, were reviewed at 1 month, 3 months and 6 months. At each of these visits (at which the doctor was assisted by a survey interviewer), patients completed a symptom questionnaire, the ESS, the TTA and TTB and the NHP. Patients randomised to group 1 brought their CPAP device to the visit to check the in-built time counter and to assess their compliance with treatment. Finally, these patients treated by CPAP had to report any problems of tolerance of the CPAP to the clinicians by telephone or at a hospital visit. These events were recorded on a case report form and any cases of discontinuation of CPAP were also recorded.

\section{Polysomnographic recording procedure and interpretation}

The same data acquisition system (CID 102*; Cidelec, Angers, France) was used in the two centres. Measurements included sleep stage (electroencephalogram, electro-oculogram, and submental electromyogram), nasal airflow (nasal cannulae) and oral airflow (oral thermistance), rib cage and abdominal wall motion (respiratory inductance plethysmography), and arterial oxygen saturation $\left(\mathrm{Sa}, \mathrm{O}_{2}\right)$. Tracheal sounds, snoring and body position was also monitored. All polysomnographic data were scored manually by an experienced observer. Sleep staging was performed according to standard criteria [29]. Apnoea was defined as cessation of airflow for $>10 \mathrm{~s}$. Hypopnoea was defined as a reduction of airflow, regardless of its amplitude, with a $>3 \%$ fall in $\mathrm{Sa}, \mathrm{O}_{2}$ or arousal [30].

In each centre, PSG recordings were interpreted by the doctor without knowing whether or not the patient was participating in the trial, and without knowledge of the data of the clinical questionnaire to avoid any classification bias.

\section{Economic calculations}

An assessment of healthcare expenditure was performed over a period of 6 months. Information was collected from patients by means of interviews conducted by a survey interviewer at each visit (1 month, 3 months and 6 months). Patients were asked to keep all prescriptions delivered between two visits. Hospitalisations (number, reason, duration), medical visits, complementary tests, biological tests and drug prescriptions were recorded, as were work accidents, home accidents or road accidents likely to be related to the disease.

Incremental cost effectiveness ratios (r) and their 95\% 
confidence intervals were calculated in the following way: $r=$ ratio of the difference of healthcare expenditure per patient between groups 1 and 2 over the difference of changes over time in terms of Epworth score, percentage of positive responses to NHP items, and scores for the six dimensions of the NHP between groups 1 and 2 .

\section{Statistical methodology}

Calculation of the number of subjects required. The variation of the NHP as a primary end-point was chosen to compare the two groups of patients. The number of subjects required was calculated on the basis of the results (coefficient of dispersion of the six dimensions of the NHP) of a recent study, to ensure a $90 \%$ chance of detecting a 13-point improvement of the "sleep" dimension (which corresponds to the capacity to detect a negative rather than a positive response to the most weakly weighted item of this dimension) with a risk of error of 5\% [18]. The calculation was based on the "sleep" dimension of the NHP, as, among the three dimensions considered to be relevant to this application to OSAS, i.e. the "emotional reactions", "energy", and "sleep" dimensions, the "sleep" dimension presented the highest coefficient of dispersion in the above mentioned study. The required sample size calculated was therefore at least 30 subjects per subgroup: 30 patients with an AHI between 10-30, immediately diagnosed and treated by CPAP, 30 patients waiting for diagnosis with an AHI between $10-30$ on the PSG performed at 6 months, 30 patients with an AHI $\geqslant 30$ immediately treated by CPAP, and finally 30 patients waiting for diagnosis, subsequently identified to suffer from OSAS with an AHI $\geqslant 30$.

Statistical tests. Results are given as the mean \pm SD. The unpaired t-test, Wilcoxon test, and Chi-squared test were used, depending on the type and distribution of the variables, to compare anthropometric and clinical variables at baseline in the two groups. The effects of treatment were analysed by using a Wilcoxon test to compare the change over time in different variables between the two groups. A p-value $<0.05$ was considered significant.

\section{Results}

\section{Baseline data}

Figure 1 shows the trial design. According to the inclusion criteria defined above, 263 patients were included in the trial, 143 randomised to group 1 and 120 randomised to group 2. A total of 61 patients in group 1 were subsequently excluded and five failed to attend the appointment for PSG. Among the patients included, 10 had an AHI $<10$ and three had a central OSAS. Among the patients with an AHI $\geqslant 10(n=125), 12$ refused home CPAP after the titration night (including five with an AHI <30) and 31 stopped CPAP before the end of the 6-month observation period (11 with an AHI <30). A total of 31 patients were subsequently excluded from group 2, 25 leaving the trial during the waiting period and PSG was subsequently not performed at 6 months, four patients had an AHI $<10$ and two patients were excluded because of the development of a serious illness (one with colon cancer and one with acromegaly). For these last two patients, a regular follow-up was not possible. The characteristics of the patients excluded are presented in table 1. In comparison with the patients included in the study, these patients were younger $(\mathrm{p}=0.0001)$, with a female over-representation $(\mathrm{p}=0.02)$.

A total of 171 patients were therefore followed throughout

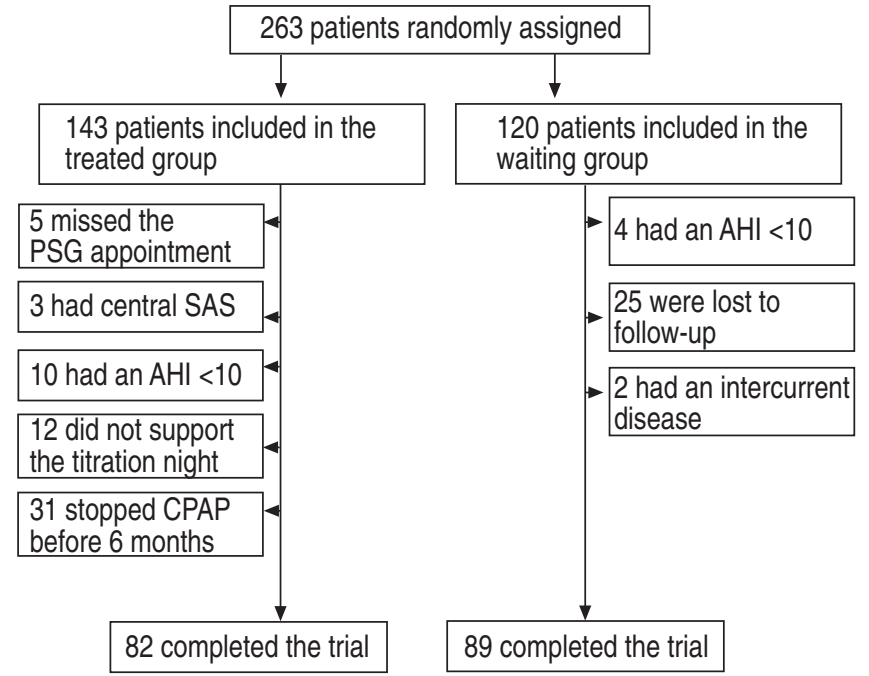

Fig. 1.-Trial design. PSG: polysomnography; AHI: apnoea/hypopnoea index; SAS: sleep apnoea syndrome; CPAP: continuous positive airway pressure.

the trial according to the predefined protocol: 82 randomised to group 1 (AHI $<30$ in 32 cases) and 89 randomised to group 2 (AHI $<30$ in 31 cases). The patients in the two groups were comparable in terms of anthropometric characteristics, severity of disease, daytime sleepiness, disorders of attention and concentration and NHP scores (table 1). No statistically significant difference was observed between the two groups in terms of concomitant chronic disease and/or concomitant medication (table 2). For all these variables, patients were not different in the two sleep centres.

\section{Continuous positive airway pressure compliance}

The mean duration of compliance with CPAP in group 1 was $5.2 \pm 2.1 \mathrm{~h}$ per night at 3 months and $4.8 \pm 2.3 \mathrm{~h}$ per night at 6 months in the subgroup of patients with an AHI $<30$, and $5.6 \pm 2.0 \mathrm{~h}$ per night at 3 months and $5.5 \pm 2.2 \mathrm{~h}$ per night at 6 months in the subgroup of patients with an AHI $\geqslant 30$, with no statistically significant difference between these two subgroups.

\section{Effects of delayed treatment}

Daytime sleepiness, cognitive functions and quality of life. Table 3 shows the effects of delayed treatment with CPAP on daytime sleepiness, cognitive functions and quality of life of OSAS patients with an AHI $\geqslant 30$. The improvement over time of these variables was significantly greater in group 1 (subjects treated immediately) than in group 2 (subjects waiting for treatment) for all variables except for TTB/TTA and for the "social isolation" dimension of the NHP.

When the same variables were analysed in the group of patients with an AHI $<30$, the difference was not significant except for the ESS score (table 4).

Healthcare consumption/expenditure. Table 5 shows the effects of delayed treatment with CPAP on healthcare consumptionexpenditure. The total healthcare expenditure per patient in group 1, treated immediately, was significantly higher than in group 2, not treated, in patients with an AHI $<30$ and in those with an $\mathrm{AHI} \geqslant 30$. This excess expenditure was no longer statistically significant when expenditure directly related to 
Table 1. - Subject characteristics according to randomisation

\begin{tabular}{|c|c|c|c|c|}
\hline \multirow{3}{*}{ Patients $n$} & \multicolumn{2}{|c|}{ No treatment } & \multicolumn{2}{|c|}{ CPAP treatment } \\
\hline & \multicolumn{2}{|c|}{120} & \multicolumn{2}{|c|}{143} \\
\hline & Excluded & Followed & Excluded & Followed \\
\hline Patients $\mathrm{n}$ & 31 & 89 & 61 & 82 \\
\hline Age yrs & $49.7 \pm 9.6$ & $52.1 \pm 8.8$ & $48.1 \pm 9.6$ & $53.8 \pm 9.7$ \\
\hline Females \% & 29.0 & 15.7 & 29.5 & 19.5 \\
\hline $\mathrm{BMI} \mathrm{kg} \cdot \mathrm{m}^{-2}$ & $29.4 \pm 5.2$ & $29.7 \pm 4.9$ & $29.1 \pm 6.4$ & $30.5 \pm 6.9$ \\
\hline $\mathrm{AHI}<30$ & & $20.2 \pm 7.5^{\#}$ & & $24.0 \pm 5^{\bullet}$ \\
\hline $\mathrm{AHI} \geqslant 30$ & & $64.4 \pm 26.2^{+}$ & & $75.9 \pm 34.9^{\S}$ \\
\hline ESS & $10.3 \pm 4.4$ & $10.3 \pm 5.1$ & $10.2 \pm 4.8$ & $9.8 \pm 4.6$ \\
\hline TTB/TTA & $2.5 \pm 0.8$ & $2.48 \pm 0.89$ & $2.7 \pm 2.1$ & $2.50 \pm 0.95$ \\
\hline Percentage of positive responses to NHP items & $20.1 \pm 12.5$ & $19 \pm 14$ & $21.7 \pm 17.3$ & $23 \pm 18$ \\
\hline \multicolumn{5}{|l|}{ NHP scores by dimension } \\
\hline Physical mobility & $12.1 \pm 14.7$ & $12.3 \pm 14.6$ & $14.4 \pm 21.8$ & $17.4 \pm 17.9$ \\
\hline Social isolation & $10.5 \pm 16.1$ & $9.6 \pm 17.6$ & $12.6 \pm 20.0$ & $11.4 \pm 20.0$ \\
\hline Pain & $12.7 \pm 20.3$ & $12.5 \pm 18.8$ & $18.6 \pm 27.5$ & $23.7 \pm 29.2$ \\
\hline Emotional reactions & $22.2 \pm 16.1$ & $20.1 \pm 20.7$ & $24.7 \pm 24.3$ & $21.7 \pm 23.5$ \\
\hline Energy & $45.4 \pm 38.2$ & $40.3 \pm 36.7$ & $47.9 \pm 38.2$ & $47.7 \pm 36.5$ \\
\hline Sleep & $31.0 \pm 28.8$ & $22.9 \pm 24.6$ & $31.1 \pm 31.3$ & $25.9 \pm 26.4$ \\
\hline
\end{tabular}

Data are presented as mean \pm SD unless otherwise stated. CPAP: continuous positive airway pressure; BMI: body mass index; AHI: apnoea/ hypopnoea index; ESS: Epworth Sleepiness Scale; TTB/TTA: ratio of trail making test B to trail making test A; NHP: Nottingham Health Profile.

\#: $n=31 ;$ ๆ: $n=32 ;{ }^{+}: n=58 ;$ \&: $n=50$.

Table 2. - Concomitant diseases and/or medications according to randomisation

\begin{tabular}{lccc}
\hline & $\begin{array}{c}\text { No } \\
\text { treatment }\end{array}$ & $\begin{array}{c}\text { CPAP } \\
\text { treatment }\end{array}$ & p-value \\
\hline Patients n & 89 & 82 & \\
Hypertension & 43.7 & 50 & 0.41 \\
$\begin{array}{l}\text { Stroke and/or peripheral } \\
\quad \text { arterial disease }\end{array}$ & 6.7 & 10.9 & 0.32 \\
Angina & 4.7 & 9.9 & \\
Diabetes & 12.8 & 19.7 & 0.19 \\
Hyperlipidaemia & 29.4 & 33.7 & 0.22 \\
Depression & 17.2 & 27.1 & 0.54 \\
Anxiolytics & 12.3 & 20.7 & 0.12 \\
Hypnotics & 7.9 & 16.1 & 0.34 \\
\hline
\end{tabular}

Data are presented as \% unless otherwise stated. CPAP: continuous positive airway pressure. treatment with CPAP was excluded from group 1. This result indicates that healthcare expenditure, over this 6-month period, was not significantly higher in patients waiting for treatment than in patients treated immediately, regardless of whether AHI was $<$ or $\geqslant 30$. This was confirmed for all types of expenditure, including general practitioner visits, specialist visits, complementary tests and drugs.

No patient reported any accidents (motor vehicle, household or work accidents) or hospitalisation during the 6-month follow-up.

\section{Incremental cost effectiveness ratios}

Table 6 summarises the results of incremental cost effectiveness ratios, i.e. the ratios of difference in healthcare expenditure per patient between groups 1 and 2 over the

Table 3.-Daytime sleepiness, cognitive functions, and quality of life before and after six months of follow-up in patients with $\geqslant 30$ apnoea and hypopnoea per hour of sleep

\begin{tabular}{|c|c|c|c|c|c|c|c|}
\hline \multirow[t]{2}{*}{ Measure } & \multicolumn{3}{|c|}{ NCPAP } & \multicolumn{3}{|c|}{ No treatment } & \multirow[t]{2}{*}{ p-value* } \\
\hline & Before & After 6 months & Difference & Before & After 6 months & Difference & \\
\hline ESS & $10.6 \pm 4.8$ & $5.6 \pm 3.4$ & $5.1 \pm 5.0$ & $10.3 \pm 5.0$ & $10.2 \pm 5.5$ & $0.2 \pm 3.4$ & $1 \times 10^{-4}$ \\
\hline TTB/TTA & $2.58 \pm 1.04$ & $2.35 \pm 0.88$ & $0.29 \pm 1.06$ & $2.43 \pm 0.96$ & $2.20 \pm 0.89$ & $0.17 \pm 1.22$ & 0.61 \\
\hline $\begin{array}{l}\text { Percentage of positive responses } \\
\text { to NHP items }\end{array}$ & $22.1 \pm 16.4$ & $9.6 \pm 12.3$ & $12.4 \pm 13.3$ & $18.5 \pm 14.0$ & $17.2 \pm 14.9$ & $0.7 \pm 10.1$ & $1 \times 10^{-4}$ \\
\hline \multicolumn{8}{|l|}{ NHP scores by dimension } \\
\hline Physical mobility & $18.2 \pm 18.8$ & $10.1 \pm 13.7$ & $8.1 \pm 16.8$ & $13.9 \pm 16.0$ & $13.2 \pm 15.5$ & $0.76 \pm 10.1$ & $6 \times 10^{-3}$ \\
\hline Social isolation & $8.3 \pm 16.8$ & $4.7 \pm 13.4$ & $3.7 \pm 17.7$ & $7.6 \pm 14.3$ & $8.1 \pm 20.1$ & $-0.6 \pm 16.5$ & 0.19 \\
\hline Pain & $22.9 \pm 28.7$ & $12.5 \pm 22.5$ & $9.7 \pm 19.0$ & $14.9 \pm 20.5$ & $13.6 \pm 20.9$ & $1.3 \pm 17.7$ & $2 \times 10^{-2}$ \\
\hline Emotional reactions & $19.9 \pm 19.6$ & $6.4 \pm 12.3$ & $13.7 \pm 18.9$ & $17.9 \pm 18.7$ & $13.4 \pm 17.5$ & $4.2 \pm 15.9$ & $7 \times 10^{-3}$ \\
\hline Energy & $47.4 \pm 35.9$ & $12.3 \pm 23.9$ & $34.7 \pm 37.2$ & $37.1 \pm 36.4$ & $41.5 \pm 54.7$ & $-4.4 \pm 47.5$ & $1 \times 10^{-4}$ \\
\hline Sleep & $24.7 \pm 26.4$ & $10.0 \pm 15.3$ & $14.6 \pm 20.2$ & $21.8 \pm 24.7$ & $22.6 \pm 26.9$ & $-0.8 \pm 13.9$ & $1 \times 10^{-4}$ \\
\hline
\end{tabular}

Data are presented as mean \pm SD unless otherwise stated. NCPAP: nasal continuous positive airway pressure; ESS: Epworth Sleepiness Scale; TTB/ TTA: ratio of trail making test B to trail making test A; NHP: Nottingham Health Profile. *: Wilcoxon test comparing the change over time (difference) observed in each group (continuous positive airway pressure (CPAP) versus waiting for CPAP). 
Table 4. -Daytime sleepiness, cognitive functions, and quality of life before and after six months of follow-up in patients with $<30$ apnoea and hypopnoea per hour of sleep

\begin{tabular}{|c|c|c|c|c|c|c|c|}
\hline \multirow[t]{2}{*}{ Measure } & \multicolumn{3}{|c|}{ NCPAP } & \multicolumn{3}{|c|}{ No treatment } & \multirow[t]{2}{*}{ p-value* } \\
\hline & Before & After 6 months & Difference & Before & After 6 months & Difference & \\
\hline ESS & $8.7 \pm 3.9$ & $6.7 \pm 3.4$ & $1.9 \pm 3.3$ & $10.1 \pm 5.2$ & $10.4 \pm 5.4$ & $-0.3 \pm 4.3$ & $2 \times 10^{-2}$ \\
\hline TTB/TTA & $2.37 \pm 0.81$ & $2.19 \pm 0.50$ & $0.20 \pm 0.90$ & $2.58 \pm 0.74$ & $2.40 \pm 0.62$ & $0.18 \pm 0.79$ & 0.93 \\
\hline $\begin{array}{l}\text { Percentage of positive responses } \\
\text { to NHP items }\end{array}$ & $26.5 \pm 20.7$ & $16.2 \pm 14.8$ & $9.7 \pm 14.0$ & $19.9 \pm 14.7$ & $15.8 \pm 15.7$ & $4.0 \pm 7.7$ & $7 \times 10^{-2}$ \\
\hline \multicolumn{8}{|l|}{ NHP scores by dimension } \\
\hline Physical mobility & $16.2 \pm 16.5$ & $14.5 \pm 15.8$ & $2.1 \pm 10.9$ & $8.9 \pm 10.9$ & $11.6 \pm 18.9$ & $-2.1 \pm 14.0$ & 0.21 \\
\hline Social isolation & $16.3 \pm 23.7$ & $7.9 \pm 19.6$ & $8.7 \pm 16.8$ & $13.3 \pm 22.3$ & $8.4 \pm 21.4$ & $4.9 \pm 22.6$ & 0.45 \\
\hline Pain & $24.8 \pm 30.4$ & $18.2 \pm 28.3$ & $6.9 \pm 25.1$ & $7.9 \pm 14.2$ & $11.1 \pm 22.6$ & $-3.6 \pm 19.2$ & $7 \times 10^{-2}$ \\
\hline Emotional reactions & $24.3 \pm 28.7$ & $9.7 \pm 16.9$ & $15.5 \pm 22.2$ & $24.2 \pm 23.6$ & $14.5 \pm 18.2$ & $9.6 \pm 12.2$ & 0.20 \\
\hline Energy & $48.2 \pm 37.8$ & $30.6 \pm 33.6$ & $17.9 \pm 36.3$ & $46.4 \pm 37.0$ & $36.4 \pm 33.9$ & $9.8 \pm 23.7$ & 0.30 \\
\hline Sleep & $27.7 \pm 26.6$ & $23.2 \pm 19.8$ & $4.9 \pm 23.1$ & $25.0 \pm 24.7$ & $23.3 \pm 23.6$ & $1.8 \pm 23.4$ & 0.58 \\
\hline
\end{tabular}

Data are presented as mean \pm SD unless otherwise stated. NCPAP: nasal continuous positive airway pressure; ESS: Epworth Sleepiness Scale; TTB/ TTA: ratio of trail making test B to trail making test A; NHP: Nottingham Health Profile. *: Wilcoxon test comparing the change over time (difference) observed in each group (continuous positive airway pressure (CPAP) versus waiting for CPAP).

Table 5. - Medical consumption per patient (in Euro) during 6 months

\begin{tabular}{|c|c|c|c|c|c|c|}
\hline & \multicolumn{3}{|c|}{ Apnoea/hypopnoea index $\geqslant 30$} & \multicolumn{3}{|c|}{ Apnoea/hypopnoea index $<30$} \\
\hline & CPAP & Waiting for CPAP & p-value* & CPAP & Waiting for CPAP & p-value* \\
\hline Patients $\mathrm{n}$ & 50 & 58 & & 32 & 31 & \\
\hline General practitioner visits & $35.1(25.9-44.2)$ & $30.7(21.3-40.1)$ & 0.24 & $28.4(18.5-38.4)$ & $34.5(18.5-50.4)$ & 0.71 \\
\hline Specialist visits & $18.7(8.0-29.4)$ & $20.4(8.0-32.9)$ & 0.91 & $37.1(20.3-54)$ & $25.8(10.2-41.4)$ & 0.32 \\
\hline Drugs & $233.6(170.6-296.5)$ & $174.3(112.8-235.7)$ & 0.06 & $257.1(158.6-355.6)$ & $198.1(108.9-287.3)$ & 0.43 \\
\hline Complementary tests & $40.9(25.4-56.4)$ & $36.27(16.8-55.7)$ & 0.21 & $64.8(31.1-98.4)$ & $38.2(22.4-54.0)$ & 0.77 \\
\hline Subtotal & $328.3(252.9-403.6)$ & $262.8(176.9-348.7)$ & 0.06 & 387.6 (257.3-517.9) & $296.6(198.2-395.1)$ & 0.47 \\
\hline CPAP treatment & 222.6 & 0 & $1 \times 10^{-4}$ & 222.5 & 0 & $1 \times 10^{-4}$ \\
\hline Total & $555.9(475.5-626.2)$ & $262.8(176.9-348.7)$ & $1 \times 10^{-4}$ & $610.1(479.8-740.4)$ & $296.6(198.2-395.0)$ & $1 \times 10^{-4}$ \\
\hline
\end{tabular}

Data are presented as mean $(95 \%$ confidence interval) unless otherwise stated. CPAP: continuous positive airway pressure. $*$ : Wilcoxon test comparing the change over time (difference) observed in each group (continuous positive airway pressure (CPAP) versus waiting for CPAP).

6-month period to difference in changes over time in terms of Epworth score, percentage of positive responses to NHP items, and scores for the six dimensions of the NHP between groups 1 and 2. It indicates that the costs per patient associated with a difference of one point decrease in the

Table 6. - Incremental cost effectiveness ratios according to apnoea/hypopnoea index (AHI)

\begin{tabular}{lcc}
\hline & AHI $\geqslant 30$ & AHI $<30$ \\
\hline $\begin{array}{l}\text { ESS } \\
\text { Percentage of positive } \\
\text { responses to NHP items }\end{array}$ & $24.3(21.0-28.6)$ & $56.3(34.4-116.6)^{\#}$ \\
NHP scores by dimension & & \\
$\quad$ Physical mobility & $39.1(29.2-57.1)$ & $75.5(52.8-116.2)$ \\
$\quad$ Social isolation & $66.6(55.8-81.4)$ & $81.5(55.0-187.4)$ \\
Pain & $34.3(30.1-39.3)$ & $29.6(22.3-40.4)$ \\
Emotional reactions & $30.3(25.4-36.8)$ & $52.7(28.4-175.1)$ \\
Energy & $7.4(6.75-8.0)$ & $38.8(22.4-95.4)^{\#}$ \\
Sleep & $18.6(15.7-22.2)$ & $97.8(83.9-112.9)^{\#}$ \\
\hline
\end{tabular}

Data are presented as mean $(95 \%$ confidence interval) of the difference in healthcare expenditure per patient between groups 1 and 2 over the 6-month period/difference in changes over time in terms of Epworth score, percentage of positive responses to NHP items and scores for the six dimensions of the NHP between groups 1 and 2. For example: expenditure (from t0 to t6) CPAP--expenditure (from t0 to t6) $_{\text {No Treatment }}$ $\left(\mathrm{ESS}_{\mathrm{t} 0}-\mathrm{ESS}_{\mathrm{t}}\right)_{\mathrm{CPAP}}-\left(\mathrm{ESS}_{\mathrm{t} 0}-\mathrm{ESS}_{\mathrm{t}}\right)_{\text {No }}$ Treatment. ESS: Epworth Sleepiness Scale; NHP: Nottingham Health Profile. \#: Incremental cost effectiveness ratio confidence intervals in the two groups did not overlap.
Epworth score, $1 \%$ decrease in positive responses to the NHP, or one point decrease in the five dimensions of the NHP (all dimensions except pain), due to rapid introduction of treatment, were lower in patients with an AHI $\geqslant 30$ than in those with an AHI $<30$. These results were confirmed by calculation of the $95 \%$ confidence intervals of the costeffectiveness ratios which, in the case of the ratios associated with a difference of one point decrease in the Epworth score, a $1 \%$ decrease in positive responses to the NHP, and a one point decrease in the score for the sleep and energy dimensions of the NHP (two most relevant dimensions of the NHP in OSAS), did not overlap in the two groups (AHI $\geqslant 30 /<30$ ).

\section{Discussion}

This randomised controlled trial confirms that a 6-month waiting time in the management of patients with severe OSAS ( $>30$ hypopnoeas/apnoeas per hour of sleep) deprives them of a significant improvement in their daytime sleepiness and quality of life during this period. The impact of delayed management in subjects with less severe OSAS (AHI <30) only concerns daytime sleepiness. Cognitive functions and healthcare expenditure are not influenced by delayed treatment regardless of the severity of the disease. Finally, incremental cost effectiveness ratios related to rapid introduction of treatment are significantly lower in the most severely ill patients. These results provide serious medical and 
economic arguments in favour of early management of the patients with more severe forms of OSAS.

Many studies have already demonstrated the positive influence of treatment by CPAP on daytime sleepiness and quality of life of patients with OSAS $[8-16,18]$. The present study confirms this result in patients with $>30$ apnoeas/ hypopnoeas per hour of sleep. As reported by MONASTERIO et al. [17], who used the same health status questionnaire (NHP), the current study demonstrated the absence of any significant effect of CPAP on the quality of life of less severe patients ( $<30$ apnoeas/hypopnoeas per hour of sleep). The results for the Epworth score were in line with those of all published studies in patients with an AHI $<30$. Only the study by MONASTERIO et al. [17] failed to demonstrate a positive effect of CPAP on daytime sleepiness in this population of mild-to-moderate OSAS. The influence of CPAP on cognitive functions, measured by TTA and TTB, has also been analysed by several authors [9-11, 13, 17, 31]. The baseline TTA and TTB scores of patients included in these studies were altered, even in less severely ill subjects (up to $125 \pm 47$ and $54 \pm 18 \mathrm{~s}$ in the series reported by MONASTERIO et al. [17] and 93.1 \pm 42.2 and $39.8 \pm 13.5 \mathrm{~s}$ in the current series, respectively). Apart from the study reported by ENGLEMAN et al. [10], no study has demonstrated a significant improvement of these tests in response to CPAP, regardless of the initial severity of the disease. The link between sleep fragmentation secondary to abnormal respiratory events and alteration of the Trail Making Test has not been clearly established [31, 32]. Although data analysis in the current study showed that cognitive functions were not influenced by delayed treatment, this finding has to be considered cautiously, since the assessment of cognitive functions was carried out by means of tests (TTA and TTB) that exclusively focus on attention and concentration. As a result, other domains of neurocognitive functions were either ignored (verbal memory, visuospatial) or roughly assessed (psychomotor speed).

Several economic evaluation studies on sleep apnoea syndrome have been recently published. Some of these studies determined healthcare expenditure in subjects with nondiagnosed OSAS [22-24], while others were devoted to analysis of the repercussions of CPAP therapy on healthcare expenditure $[19,21]$. In the case-control study by KAPUR et al. [22], the authors found that the mean direct medical cost measured over the year preceding the diagnosis was two-fold higher in OSAS patients (US\$2,720) compared with age and sexmatched controls (US\$1,384) and that a significant difference persisted after adjustment for concomitant chronic diseases. They also showed that healthcare expenditure during the 1-yr study period was independently related to the severity of OSAS measured by the AHI. OTAKE et al. [24], in Canada, using the same study design, showed that OSAS patients received a greater number of prescribed drugs per year and a greater number of days of treatment with prescribed drugs than non-OSAS controls. In a telephone interview survey, OHAYON et al. [23] showed that $31 \%$ of subjects reporting breathing pauses had sought medical help six times or more in the past 12 months compared with only $11.9 \%$ of nonsnorers $(\mathrm{p}=0.001)$. ВАНАМмАм et al. [19] showed that the difference in healthcare expenditure, in terms of medical visits and hospitalisations, between OSAS patients treated for $2 \mathrm{yrs}$ compared to non-OSAS controls was less than the difference in healthcare expenditure between these same patients and the same controls before treatment $(\mathrm{C} \$ 174 \pm 32.4$ per yr versus C\$260土35.7). In a similar study, GEORGE [21] showed that patients with untreated OSAS had significantly more road accidents (and therefore more related healthcare expenditure), during the 3 yrs before starting treatment, than matched controls of the general population $(0.18$ versus 0.06 accidents per driver per year), and this difference disappeared during the 3 yrs after introduction of CPAP (0.06 versus 0.06$)$. In the current study, no statistically significant difference was detected between the two groups, treated and waiting for treatment, in patients with either $<30$ or $>30$ apnoeas-hypopnoeas per hour of sleep. A tendency of higher medication consumption was observed in subjects under CPAP compared with subjects waiting for treatment, and this tendency was more marked in the patients with more severe OSAS (€233.6 versus $€ 174.3$ in patients with $\mathrm{AHI} \geqslant 30, \mathrm{p}=0.06$; $€ 257.1$ versus $€ 198.1$ in patients with $\mathrm{AHI}<30, \mathrm{p}=0.43$ ). As it is reported in the literature, healthcare costs are high in untreated OSAS, but the results from the current study show that these costs do not necessarily decrease after a short period of time with CPAP. These findings attenuate the conclusion of BАНАMMAM et al. [19] according to which "early diagnosis and treatment of patients with OSAS would not be a burden on healthcare system, but instead may result in significant cost saving". Several hypotheses can be proposed to explain these results. First, patients included in the current study, under CPAP or waiting for treatment, had all decided to consult for management of their OSAS, and had been informed by the nursing staff about the importance of a healthy lifestyle and the potential consequences of OSAS (chronic diseases not yet detected or treated) on their state of health. This increased awareness may have had a greater impact on healthcare expenditure of patients treated immediately, as they were sure, without having to wait 6 months that they suffered from OSAS. Secondly, the follow-up period of the current study was 6 months (the usual waiting time for a diagnosis of OSAS). As the two groups of patients (treated and waiting for treatment) were comparable in terms of chronic diseases at the beginning of the study, it may be hypothesised that there was no reason why the frequency of specialist visits and drug prescriptions related to the management of these diseases should change over such a short period of time.

To the authors' knowledge, this is the first study to compare, under the conditions of a randomised clinical trial, the healthcare expenditure of patients treated for OSAS and those waiting for treatment. This study may present a number of limitations. First, it was not placebo-controlled. However, the objective of this study was not to take into account the placebo effect, but to construct a "pragmatic" study protocol in order to compare, all other things being equal, the usual management (6-month waiting time with health and dietary advice at the first visit, consultations at 1,3 and 6 months) with optimal management (i.e. immediate diagnosis and treatment). Secondly, it is debateable whether patients who report daytime sleepiness interfering with car driving, patients with unstable angina and, in general, those with an indication for an emergency treatment, which are more likely to generate costs, should be excluded from the study. However, it is to be stressed that routine practice results in immediate management of the patients with an indication for emergency treatment. As a result, inclusion of these patients in the study would have distorted the findings with respect to the defined objectives i.e. to evaluate the clinical, economic and quality of life consequences of a 6-month waiting time, as observed under usual management conditions. Thirdly, as in studies that compare medical outcomes in terms of economic parameters, such as cost-effectiveness, statistical significance and quantitative importance of the observed differences could also be questioned in the current study. However, there is no immediate answer to such a question of whether an intervention (here the management of OSAS patients without any delay) offers sufficient value for money to warrant resources being reallocated to it is a collective decision requiring the input of public preferences about the relative importance of alternative therapies and health benefits [33]. Fourthly, randomised patients who refused or stopped CPAP 
in group 1 or who did not comply with the intervention protocol in group 2 were excluded from the analysis. It may be argued that this could bias the clinical and economic outcomes. Unfortunately, these patients were lost to followup, which leaves the dilemma of the intention-to-treat principle. The necessary clinical and economic data are therefore not available for these patients and certain hypotheses need to be proposed. In the group of treated patients, it may be hypothesised that the inclusion of noncompliant patients in the analysis would decrease the difference of improvement of ESS and NHP measured after 6 months. However, there is no reason to suppose that these noncompliant patients consumed more healthcare resources. In the group of nontreated patients, it may be hypothesised that the inclusion of patients lost to follow-up would not alter the absence of any significant variation of ESS and NHP over the study period. In terms of costs, it may be hypothesised that nontreated patients not complying with the protocol consumed the same resources as compliant patients (apart from follow-up visits). Overall, if the two groups are compared in economic terms, the conclusions probably remain the same. However, the incremental cost effectiveness ratios are probably increased (smaller difference of efficacy delta between the two groups for the same absence of cost difference), although the proportions cannot be predicted in patients with $<30$ and $>30$ apnoeas per hour. Fifthly, the economic results of this study could possibly be considered to be dependent on the healthcare setting in which the patients were managed and could therefore not be generalised to other settings. This would be true if the analysis was based on the absolute figures obtained for the various types of expenditure. However, when the analysis is based on comparative values, as in the current study, in which patients under CPAP were compared with patients waiting for treatment, this criticism is unfounded. This comment also applies to analysis of the overlap of $95 \%$ confidence intervals for incremental cost effectiveness ratios in the two groups. If the costs of medical visits, drugs, etc. in the various healthcare settings were increased or decreased by $10 \%, 20 \%, 50 \%$ or more, the absolute values obtained for these ratios would be modified, but the $95 \%$ confidence intervals for incremental cost effectiveness ratios would vary in the same proportions and interpretation of the results in terms of overlap of confidence intervals would remain unchanged.

In conclusion, a 6 month delay in treating patients with obstructive sleep apnoea syndrome does not result in any additional health related cost, regardless of the severity of disease. However, in a context of allocation of scarce resources, there is economic evidence for treating patients with an apnoea/hypopnoea index of $\geqslant 30$ first, since incremental costs are lower for these patients.

\section{References}

1. Fleury B, Rakotonanahary D, Tehindrazanarivelo AD, Hausser-Hauw C, Lebeau B. Long-term compliance to continuous positive airway pressure therapy (nCPAP) set up during a split-night polysomnography. Sleep 1994; 17 : 512-515.

2. McArdle N, Grove A, Devereux G, Mackay-Brown L, Mackay T, Douglas NJ. Split-night versus full-night studies for sleep apnoea/hypopnoea syndrome. Eur Respir $J$ 2000; 15: 670-675.

3. Portier F, Portmann A, Czernichow P, et al. Evaluation of home versus laboratory polysomnography in the diagnosis of sleep apnoea syndrome. Am J Respir Crit Care Med 2000; 162: 814-818.

4. Gagnadoux F, Pelletier-Fleury N, Philippe C, Rakotonanahary
D, Fleury B. Home unattended vs hospital telemonitored polysomnography in suspected obstructive sleep apnoea syndrome: a randomized crossover trial. Chest 2002; 121: 753-758.

5. Pelletier-Fleury N, Lanoe JL, Philippe C, Gagnadoux F, Rakotonanahary D, Fleury B. Economic studies and "technical" evaluation of telemedicine: the case of telemonitored polysomnography. Health Policy 1999; 49: 179-194.

6. Chervin RD, Murman DL, Malow BA, Totten V. Costutility of three approaches to the diagnosis of sleep apnoea: polysomnography, home testing, and empirical therapy. Ann Intern Med 1999; 130: 496-505.

7. Reuven H, Schweitzer E, Tarasiuk A. A cost-effectiveness analysis of alternative at-home or in-laboratory technologies for the diagnosis of obstructive sleep apnoea syndrome. Med Decis Making 2001; 21: 451-458.

8. Ballester E, Badia JR, Hernandez L, et al. Evidence of the effectiveness of continuous positive airway pressure in the treatment of sleep apnoea/hypopnoea syndrome. Am J Respir Crit Care Med 1999; 159: 495-501.

9. Barbe F, Mayoralas LR, Duran J, et al. Treatment with continuous positive airway pressure is not effective in patients with sleep apnoea but no daytime sleepiness. A randomized, controlled trial. Ann Intern Med 2001; 134: $1015-1023$.

10. Engleman HM, Martin SE, Deary IJ, Douglas NJ. Effect of continuous positive airway pressure treatment on daytime function in sleep apnoea/hypopnoea syndrome. Lancet 1994; 343: 572-575.

11. Engleman HM, Martin SE, Deary IJ, Douglas NJ. Effect of CPAP therapy on daytime function in patients with mild sleep apnoea/hypopnoea syndrome. Thorax 1997; 52: 114-119.

12. Engleman HM, Martin SE, Kingshott RN, Mackay TW, Deary IJ, Douglas NJ. Randomised placebo controlled trial of daytime function after continuous positive airway pressure (CPAP) therapy for the sleep apnoea/hypopnoea syndrome. Thorax 1998; 53: 341-345.

13. Engleman HM, Kingshott RN, Wraith PK, Mackay TW, Deary IJ, Douglas NJ. Randomized placebo-controlled crossover trial of continuous positive airway pressure for mild sleep Apnoea/Hypopnoea syndrome. Am J Respir Crit Care Med 1999; 159: 461-467.

14. Jenkinson C, Davies RJ, Mullins R, Stradling JR. Comparison of therapeutic and subtherapeutic nasal continuous positive airway pressure for obstructive sleep apnoea: a randomised prospective parallel trial. Lancet 1999; 353: 2100-2105.

15. Montserrat JM, Ferrer M, Hernandez L, et al. Effectiveness of CPAP treatment in daytime function in sleep apnoea syndrome: a randomized controlled study with an optimized placebo. Am J Respir Crit Care Med 2001; 164: 608-613.

16. Redline S, Adams N, Strauss ME, Roebuck T, Winters M, Rosenberg C. Improvement of mild sleep-disordered breathing with CPAP compared with conservative therapy. Am J Respir Crit Care Med 1998; 157: 858-865.

17. Monasterio C, Vidal S, Duran J, et al. Effectiveness of continuous positive airway pressure in mild sleep apnoeahypopnoea syndrome. Am J Respir Crit Care Med 2001; 164: 939-943.

18. Meslier N, Lebrun $\mathrm{T}$, Grillier-Lanoir V, et al. A French survey of 3,225 patients treated with CPAP for obstructive sleep apnoea: benefits, tolerance, compliance and quality of life. Eur Respir J 1998; 12: 185-192.

19. Bahammam A, Delaive K, Ronald J, Manfreda J, Roos L, Kryger MH. Healthcare utilization in males with obstructive sleep apnoea syndrome two years after diagnosis and treatment. Sleep 1999; 22: 740-747.

20. Findley LJ, Suratt PM. Serious motor vehicle crashes: the cost of untreated sleep apnoea. Thorax 2001; 56: 505.

21. George CF. Reduction in motor vehicle collisions following treatment of sleep apnoea with nasal CPAP. Thorax 2001; 56: $508-512$. 
22. Kapur V, Blough DK, Sandblom RE, et al. The medical cost of undiagnosed sleep apnoea. Sleep 1999; 22: 749-755.

23. Ohayon MM, Guilleminault C, Priest RG, Caulet M. Snoring and breathing pauses during sleep: telephone interview survey of a United Kingdom population sample. $B M J$ 1997; 314: 860-863.

24. Otake K, Delaive K, Walld R, Manfreda J, Kryger MH. Cardiovascular medication use in patients with undiagnosed obstructive sleep apnoea. Thorax 2002; 57: 417-422.

25. Pack AI, Gurubhagavatula I. Economic implications of the diagnosis of obstructive sleep apnoea. Ann Intern Med 1999; 130: $533-534$.

26. Johns MW. A new method for measuring daytime sleepiness: the Epworth sleepiness scale. Sleep 1991; 14: 540-545.

27. Adams N, Strauss M, Schluchter M, Redline S. Relation of measures of sleep-disordered breathing to neuropsychological functioning. Am J Respir Crit Care Med 2001; 163: 16261631.

28. Bucquet D, Condon S, Ritchie K. The French version of the Nottingham Health Profile. A comparison of items weights with those of the source version. Soc Sci Med 1990; 30: 829835 .

29. Reschaffen AKA. A manual of standardized terminology: techniques and scoring system for sleep stages of human subjects. Los Angeles, Brain Information Service, 1968.

30. Task Force of the American Sleep Disorders Association. EEG arousals: scoring rules and examples: a preliminary report from the Sleep Disorders Atlas Task Force. Sleep 1992; 15: 173-184.

31. Henke KG, Grady JJ, Kuna ST. Effect of nasal continuous positive airway pressure on neuropsychological function in sleep apnoea-hypopnoea syndrome. A randomized, placebocontrolled trial. Am J Respir Crit Care Med 2001; 163: 911-917.

32. Kim HC, Young T, Matthews CG, Weber SM, Woodward AR, Palta M. Sleep-disordered breathing and neuropsychological deficits. A population-based study. Am J Respir Crit Care Med 1997; 156: 1813-1819.

33. O'Brien BJ, Drummond MF. Statistical versus quantitative significance in the socioeconomic evaluation of medicines. Pharmacoeconomics 1994; 5: 389-398. 